Editorial

\title{
Acknowledgement to Reviewers of Arts in 2019
}

Arts Editorial Office

MDPI, St. Alban-Anlage 66, 4052 Basel, Switzerland

Published: 17 January 2020

The editorial team greatly appreciates the reviewers who have dedicated their considerable time and expertise to the journal's rigorous editorial process over the past 12 months, regardless of whether the papers are finally published or not. In 2019, a total of 152 papers were published in the journal, with a median time to first decision of 24 days and a median time from submission to publication of 63 days. The editors would like to express their sincere gratitude to the following reviewers for their generous contribution in 2019:

\begin{tabular}{ll} 
Abiola, Ofosuwa & Bergamasco, Massimo \\
Adzhiev, Valery & Bergera, Inaki \\
Ahmedien, Diaa & Bevan, Alex \\
Alexandridis, Annetta & Bevan, George \\
Alexandridou, Alexandra & Bialek, Goshka \\
Allen, Ruth & Bingham, Adam \\
Alsop, Roger & Bingham, Adam \\
Alvarez, Mariola & Binnie, Mari Rodriguez \\
Anderson, Joel & Bishop, Mark \\
Anderson, Suse & Blake, Sarah H. \\
Andreallo, Fiona & Bläsing, Bettina \\
Andrew, Nell & Blaylock, Sara \\
Andrianou, Dimitra & Blom, Kati \\
Angliker, Erica & Blough, Karen \\
Anz, Craig & Blow, Mike \\
Apodaca, Paul & Blumenthal-Barby, Martin \\
Armstrong, Stuart & Bodner, Neta \\
Arrigoni, Gabi & Boldrick, Stacy \\
Ashmore, Rupert & Broadhead, Sam \\
Badu-Younge, Zelma & Brokalaki, Zafeirenia \\
Baines, Jenny & Brooker, Caelli \\
Baker, Catherine & Brown, Benita \\
Barham, Jeremy & Brown, Elizabeth \\
Bates, Alex & Brown, Ian \\
Batey, Jackie & Brubaker, Leslie \\
Bautz, Amy & Bunning, Katy \\
Belarde-Lewis, Miranda & Burns, Emily \\
Belden-Adams, Kris & Burns, Kara \\
Bell, David & Burridge, Stephanie \\
Bell, John & Butler, Rex \\
Benamou, Catherine L. & Caines, Rebecca \\
Bennett, Alexis & Calvert, Sheena \\
Bennett, Danielle Smotherman & Carter, Selene \\
Ben-Tal, Oded & Cather, Kirsten \\
Berg, Nancy E. & Chambers, Ruth \\
& \\
\hline
\end{tabular}


Chance, Frank L.

Changfoot, Nadine

Chapman, Sarah

Chiodi, Stefano

Chivers, Morgan

Clottes, Jean

Coates, Jennifer

Codell, Julie

Colangelo, Dave

Colin, Sandrine

Collard, Judith

Collier, Mike

Conradi Chavez, Ina

Corcoran, Lorelei $\mathrm{H}$.

Corrie, Rebecca

Corrieri, Augusto

Costa, Fabrizio

Covaci, Alexandra

Crang, Mick A.

Crenshaw, Melissa

Cressier, Patrice

Cutller, Vanessa

D'Ambra, Eve

Damian, Carol

Dark, Ken

Davies, Glenys

Déguernel, Ken

Dekel, Tal

Delhaye, Christine

Desser, David

Dexl, Carmen

Diack, Heather

Dickey, Stephanie

Dobrez, Patricia

Doig, Allan

Dolezalek, Isabelle

Donati, Alessandra

Donger, Simon

Donohue, A.A.

Doyle, Jessica

Draycott, Catherine M.

Dunn, Stuart

Echevarría López, Gori-Tumi

Eiroa, Jorge

Elia, Ricardo J.

Eliassi, Barzoo

Ellis-Geiger, Robert

Farooq, Yasmin Ghazala

Fenner, Felicity

Findlay, Elisabeth

Fleming, Alison

Florance, Caren
Foster, Susan

Foukara, Lavinia

Fragaki, Eleni

Franko, Mark

Franks, Hallie

Fred Cahir, David

Fuchsgruber, Lukas

Fusco, Maria

García, Jorge Tomás

Garcia-Velasco, Rodrigo

Gardner, Julian

Gardner, Sally

Garfield, Rachel

Gasanova, Svetlana

Gatt, Michael

Gavan, Jane

Gee, Malcolm

Gere, Charlie

Geva, Anat

Geymonat, Ludovico

Gill, Satinder

Gillett, John

Glade-Wright, Robyn

Glynn, Dominic

Gold, Alexandra

Gotman, Kelina

Graddy, Kathryn

Graham, Katherine

Grainge, Paul

Grant, Katrina

Greated, Marianne

Greenleaf, Eric A.

Greet, Michele

Griffiths, Laura

Gruber, Samuel

Gunn, Robert (Ben)

Gupta, Mani Das

Hafsteinsson, Sigurjón Baldur

Halart, Sophie

Halfyard, Janet K

Hall, Mark

Halperin, Dalia-Ruth

Hamby, Louise

Hampson, Jamie

Hannigan, Shelley

Harden, Alastair

Harding, Richard

Harman, Mary

Harris, Chris

Harty, Kim

Havsteen-Franklin, Dominik

Hawley, Elizabeth 
Hearne, James

Heldt, Guido

Hendrick, Catharina

Henebry-Deleon, Lourdes

Herring, Edward

Hertz, Paul

Hertzmann, Aaron

Hijmans, Steven

Hllman, Roger

Ho, Angela

Hodgson, Douglas J.

Hölling, Hanna

Honig, Elizabeth

Hoover, Elizabeth M.

Horton, Jessica

Hostetler, Brad

Hughes, Helen

Huss, Ephrat

Hutchinson, Elizabeth

Ikram, Salima

Ireland, Dave

Iversen, Gunnar

Jaakkola, Maarit

Jackson Williams, Kelsey

Jacoby, Alexander

James, Diana

James-Chakraborty, Kathleen

Janik, Liliana

Jaritz, Gerhard

Johnson, Benjamin

Jones, Jason C.

Jones, Nathaniel

Jones, Owain

Jones, Philip

Jordan, Kimberleigh

Jordano, Ángeles

Judge, Mark

Kappenberg, Claudia

Kehoe, Marsely

Kellum, Barbara

Kelly, Jessica

Kemper, Steven

King, Edward

Kitsnik, Lauri

Klaniczay, Gábor

Klein, John

Klein, Rudolf

Knees, Peter

Knoblauch-O'Neal, Christine

Kramer, Karen

Kravtsov, Sergey

Krinsky, Carol
Kristensen, Troels Myrup

Kubo, Yutaka

Kupietzky, Allison

Kurz, Joshua James

Kvapil, Lynne

Laferriere, Carolyn

Lamm, Kimberly Kay

Lang, Sabine

Langridge-Noti, Elizabeth

Laumond, Jean-Paul

Lawrenson, Anna

Ledent, Gérald

Lee, Christina

Lefebvre Sell, Naomi

Lefebvre, Antoine

Lefebvre, Corinne

Leipert, Trent Clark

Lerner, L. Scott

Leung, Isaac

Lewis, Diane Wei

Lewis, Sian

Lifshitz, Isaac

Liggett, Susan

Lioret, Alain

Löfgren, Eva

López, José C. Carvajal

Lovell, Margaretta

Lowish, Susan

Lu, David

Lübbren, Nina

Lucherini, Vinni

Lupi, Livia

Luvaas, Brent

Ma, Ran

Mannack, Thomas

Marcus Moore, Marcus

Marenko, Betti

Marks, Laura U

Martin, Charles

Martin, Rose

Martínez Jiménez, Javier

Martinon, Jean-Paul

Maulsby, Lucy

Maxwell, Kathleen

Mazzone, Marian

McCaw, Dick

McClanahan-Simmons, Angela

McCleary, Kristen

McEntee, Joy

McNiven, Timothy

Mencía, María

Merwood-Salisbury, Joanna 
Mes, Thomas

Meyer, Mati

Mickūnaitè, Giedrè

Mitchell, Joanne

Molina Figueras, Joan

Moore, Abigail Harrison

Morgan, Emily

Morgenthaler, Hans R.

Morreale, Fabio

Morris, Simon

Morse, Nicole

Moure Cecchini, Laura

Moys, Jeanne-Louise

Murphy, Kayleigh

Narváez Torregrosa, Daniel C.

Nichols, Marden Fitzpatrick

Nicoll, Fiona

Nugent, Ann

O'Rawe, Des

O'Reilly, Sean

Ortal-Paz, Saar

Osborne, Robin

Ottarsdottir, Unnur

Page, Michael

Pardo, Bryan

Peck, Julia

Pérez, Diana Rodríguez

Peruzzi, Bice

Petersén, Moa

Pfuntner, Laura

Pinxit, Vaughn

Pitman, Thea

Plantzos, Dimitris

Pocock, Celmara

Podzorski, Patricia V.

Pratt, Stephanie

Prichard, Franz

Prince, Jocelyne

Quintero, Henry

Rajendran, Lakshmi Priya

Ravetz, Amanda

Rawle, Steven

Rawlinson, Mark

Recht, Laerke

Revill, George

Reynolds, Kendra

Richardson, Craig

Richardson, Martin

Richmond, Susan

Rickard, Jolene

Riggs, Christina

Ripatti, Anna
Ristow, Sebastian

Rix, Brenda

Robertson, Frances

Rogers, Kathleen

Rolls, Mitchell

Rosa, Luis Othoniel

Roscino, Carmela

Ross, Alexandra

Rossato, Luca

Roush, Paula

Ruprecht, Lucia

Rutgers, Leonard

Ryan, Allan J.

Sachs, Emma

Saint-Raymond, Léa

Saka, Gizem

Samuel, Jamuna

Savvopoulos, Kyriakos

Sayers, Esther

Schneller, Tom

Schultz, Deborah

Schuster, Kristen

Schwall, Elizabeth

Scott, Sarah

Seccombe, Erica

Seelow, Atli Magnus

Shapiro, Amy Rebecca

Shimon, Dar

Sicchio, Kate

Silverstein, Shayna

Slatter, Ruth

Slevin, Tom

Slivka, Kevin

Smith, Amy

Smith, Benjamin

Smith, Olga

Smith, Sarah

Soila, Tytti

Solomon, Virginia

Solovieva, Olga

Soon-Hwa, Oh

Squire, Michael

Srinivasan, Anjali

Stammers, Thomas

Stanciu, Cristina

Stara, Alexandra

Steinbock, Eliza

Stewart, David

Stiefel, Barry

Stiles, Emily

Streit, Jessica

Streit, Katharina 
Sutters, Justin P.

Swalwell, Melanie

Swanz, Jonathan

Swift, Ellen

Symko, Riva

Tanner, Simon

Taylor, Paul

Thoma, Andrea

Thomas, Beth A.

Thomason, Allison Karmel

Thornton, Sybil

Tolar, Tanja

Toloudi, Zenovia

Tsouparopoulou, Christina

Tully, Caroline

Vail, Gabrielle

Van De Moortel, Aleydis

Van Den Hoven, Carina

Vanderstaay, Lara

Verhoeff, Nanna

Vermeylen, Filip

Veszprémi, Nóra

Vettel-Becker, Patricia

Voelker, Emily

Vuong, Lea

Wagner, Barbara

Ware, Ianto

Watson, Mark

Watts, Emma
Weir, Simon

Weiss, Zeev

Wells, Liz

Wesley, Daryl

Westphalen, Stephan

Westwood, Jill

White, Anthony

Whiteman, Stephen

Whitewright, Julian

Whitley, David

Whitley, James

Whyte, William

Wickstead, Helen

Willis, Steven

Willmott, Cory

Wilson, Andrew

Wimmer, Dorothee

Wodak, Josh

Woldemikael, Tekle

Wolfe, Edith

Woods, Penelope

Wyatt, Victoria

Xambó, Anna

Yao, Sijia

Young, Diana

Zarobell, John

Zeng, Benxiang

Ziółkowski, Grzegorz

Zuanni, Chiara

(C) 2020 by the author. Licensee MDPI, Basel, Switzerland. This article is an open access article distributed under the terms and conditions of the Creative Commons Attribution (CC BY) license (http://creativecommons.org/licenses/by/4.0/). 